Biotechnology for Biofuels

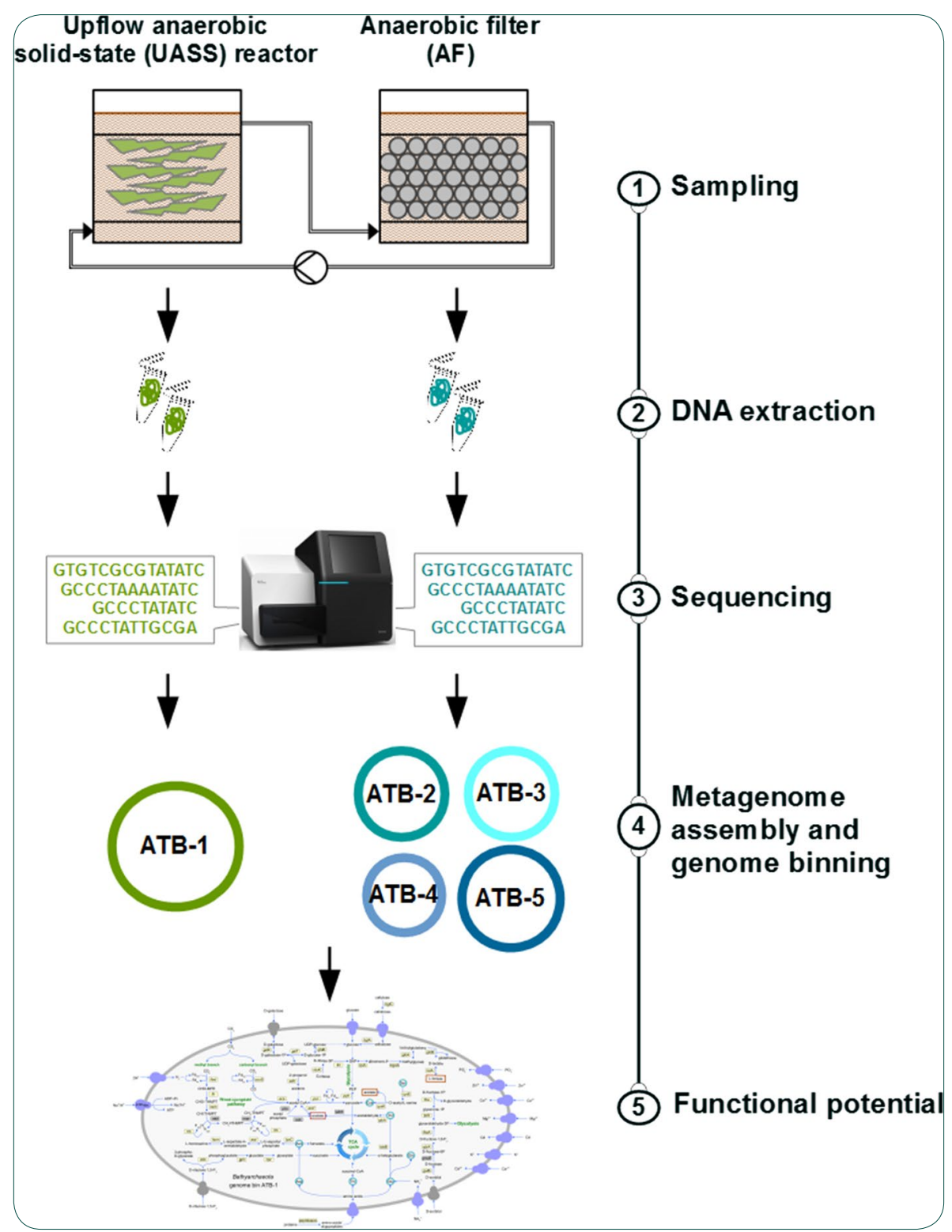

\title{
Characterization of Bathyarchaeota genomes assembled from metagenomes of biofilms residing in mesophilic and thermophilic biogas reactors
}

Maus et al. 


\title{
Characterization of Bathyarchaeota genomes assembled from metagenomes of biofilms residing in mesophilic and thermophilic biogas reactors
}

Irena Maus ${ }^{1,2 \dagger}$, Madis Rumming ${ }^{2,3 \dagger}$, Ingo Bergmann ${ }^{1}$, Kathrin Heeg ${ }^{1}$, Marcel Pohl ${ }^{4}$, Edith Nettmann ${ }^{5}$, Sebastian Jaenicke ${ }^{6}$, Jochen Blom ${ }^{6}$, Alfred Pühler ${ }^{2}$, Andreas Schlüter ${ }^{2 \ddagger}$, Alexander Sczyrba ${ }^{2,3 \ddagger}$ and Michael Klocke ${ }^{1 * \neq}$ (1)

\begin{abstract}
Background: Previous studies on the Miscellaneous Crenarchaeota Group, recently assigned to the novel archaeal phylum Bathyarchaeota, reported on the dominance of these Archaea within the anaerobic carbohydrate cycle performed by the deep marine biosphere. For the first time, members of this phylum were identified also in mesophilic and thermophilic biogas-forming biofilms and characterized in detail.

Results: Metagenome shotgun libraries of biofilm microbiomes were sequenced using the Illumina MiSeq system. Taxonomic classification revealed that between 0.1 and $2 \%$ of all classified sequences were assigned to Bathyarchaeota. Individual metagenome assemblies followed by genome binning resulted in the reconstruction of five metagenome-assembled genomes (MAGs) of Bathyarchaeota. MAGs were estimated to be $65-92 \%$ complete, ranging in their genome sizes from 1.1 to $2.0 \mathrm{Mb}$. Phylogenetic classification based on core gene sets confirmed their placement within the phylum Bathyarchaeota clustering as a separate group diverging from most of the recently known Bathyarchaeota clusters. The genetic repertoire of these MAGs indicated an energy metabolism based on carbohydrate and amino acid fermentation featuring the potential for extracellular hydrolysis of cellulose, cellobiose as well as proteins. In addition, corresponding transporter systems were identified. Furthermore, genes encoding enzymes for the utilization of carbon monoxide and/or carbon dioxide via the Wood-Ljungdahl pathway were detected.
\end{abstract}

Conclusions: For the members of Bathyarchaeota detected in the biofilm microbiomes, a hydrolytic lifestyle is proposed. This is the first study indicating that Bathyarchaeota members contribute presumably to hydrolysis and subsequent fermentation of organic substrates within biotechnological biogas production processes.

Keywords: Archaea, Bathyarchaeota, Biomass conversion, Anaerobic digestion, Biomethanation, Hydrolysis, Metabolic pathway reconstruction, Metagenome-assembled genomes, Genome binning

\footnotetext{
*Correspondence: mklocke@atb-potsdam.de

†'Irena Maus and Madis Rumming contributed equally to this work

${ }^{\ddagger}$ Andreas Schlüter, Alexander Sczyrba and Michael Klocke contributed

equally to this work

${ }^{1}$ Dept. Bioengineering, Leibniz Institute for Agricultural Engineering

and Bioeconomy (ATB), Max-Eyth-Allee 100, 14469 Potsdam, Germany

Full list of author information is available at the end of the article
}

(c) The Author(s) 2018. This article is distributed under the terms of the Creative Commons Attribution 4.0 International License (http://creativecommons.org/licenses/by/4.0/), which permits unrestricted use, distribution, and reproduction in any medium, provided you give appropriate credit to the original author(s) and the source, provide a link to the Creative Commons license, and indicate if changes were made. The Creative Commons Public Domain Dedication waiver (http://creativecommons.org/ publicdomain/zero/1.0/) applies to the data made available in this article, unless otherwise stated. 


\section{Background}

The bioconversion of biomass to biogas by anaerobic digestion (AD) is a process commonly found in nature which is performed by highly diverse and dynamic microbial communities. In the break-down cascade of macromolecular compounds, methanogenesis is the last step conducted exclusively by methanogenic Archaea of the phylum Euryarchaeota.

The structure and development of biomass-degrading microbial communities residing in biogas plants and, in particular, of the participating methanogenic archaeal species have been intensively studied [1-4]. Hydrogenotrophic Archaea utilizing $\mathrm{H}_{2}$ and $\mathrm{CO}_{2}$ often dominate the archaeal sub-communities in biogas-producing systems, while the acetoclastic and methylotrophic methanogens are less abundant $[3,5] . \mathrm{H}_{2} / \mathrm{CO}_{2}$ as well as acetate and other volatile fatty acids are provided by various fermentative bacteria predominantly affiliated with the classes Clostridia and Bacteroidia [2, 4, 6]. However, metagenome studies addressing biogas-producing microbial community characterization reported on a huge fraction of sequences that cannot be classified to higher taxonomic ranks suggesting that, for the most part, the microbial species present in biogas microbiomes are so far unknown $[4,7]$.

On the other hand, the non-cultivable fraction of biogas-producing microbial communities becomes accessible even by applying metagenome assemblies combined with binning methods enabling the identification of novel and, hence, metabolically uncharacterized species $[8$, 9]. Using this strategy, Evans and colleagues [10] were able to recover two metagenome-assembled genomes (MAGs, denominated as BA1 and BA2) of the phylum Bathyarchaeota from a deep aquifer habitat within the Surat Basin (Australia). The proposed phylum Bathyarchaeota of the domain Archaea represents an evolutionary diverse group of microorganisms (previously denominated as Miscellaneous Crenarchaeotal Group, MCG) supposed to be widespread in nature [11-13]. In particular, the organic-rich sediments of the White Oak River estuary (North Carolina, USA) were described to be abundant in uncultured Archaea, especially members of the phylum Bathyarchaeota [12, 14, 15]. Studies on Bathyarchaeota metabolic function in situ via stable carbon isotope probing of the sediment archaeal community suggested that they assimilate organic carbon sources including acetate, glycine or urea, or complex biopolymers such as lipids, proteins, and the algal lipid/ pigment extract in their sediment habitat [16]. A recent study by He and colleagues [17] indicated that Bathyarchaeota also have the potential to fix inorganic carbon in the form of $\mathrm{CO}_{2}$ to produce acetate, an important substrate for other sediment residents such as methanogenic
Archaea or heterotrophic Bacteria. Moreover, based on the metabolism reconstructed from the MAG datasets, Evans and colleagues [10] suggested that BA1 and BA2, originating from microbial biomass from filtered waters within the Surat Basin (Queensland, Australia), are capable of methylotrophic methanogenesis indicating that methane metabolism also may exist outside the phylum Euryarchaeota.

This study focusses exclusively on the identification of Bathyarchaeota members in exemplary biotechnological $\mathrm{AD}$ processes and the analysis of their putative role during biomethanation of crop biomass and residues. Since previous studies reported on the abundance of Bathyarchaeota in natural environments, it was also of importance for this study to determine the abundance of this archaeal group in biogas reactor systems and to analyze whether standard reactor operating parameters might affect their occurrence. For this purpose, the metagenomes of different biomass-degrading and biogas-producing biofilm microbiomes obtained from different mesophilic $\left(37^{\circ} \mathrm{C}\right)$ and thermophilic $\left(55^{\circ} \mathrm{C}\right)$ two-phase, two-stage laboratory-scale biogas reactor systems consisting each of hydrolysis fermenters and anaerobic filters were sequenced.

Metagenome assemblies followed by a binning approach resulted in the identification of five Bathyarchaeota MAGs which were further analyzed in detail. These MAGs represent the first Bathyarchaeota members that have been identified in biogas-producing reactor systems so far.

\section{Methods}

\section{Set-up, operation, and sampling of biofilms}

from two-phase, two-stage laboratory-scaled biogas fermenter systems

Three laboratory-scaled experimental biogas fermenter systems were sampled. As inocula for fermenter startup, digestates and/or process liquids from previous $\mathrm{AD}$ experiments were used. System 1 was a thermophilic $\left(55^{\circ} \mathrm{C}\right)$ two-phase, two-stage reactor system consisting of an upflow anaerobic solid-state (UASS) reactor digesting wheat straw as sole substrate and a downstream packed bed anaerobic filter (AF) with working volumes of 39 and $30 \mathrm{~L}$, respectively [18]. Samples for microbial DNA extraction and subsequent metagenome sequencing were taken from the wheat straw digestate in the UASS to obtain the digestate-attached cellulolytic/hydrolytic biofilm, at day 160 of reactor operation and an organic loading rate (OLR) of $8 \mathrm{~g}$ volatile substances (VS) $\mathrm{L}^{-1}$ day $^{-1}$. System 2 was constructed similar to system 1 but with a working volume of $27 \mathrm{~L}$ for the UASS and $22 \mathrm{~L}$ for the AF [19]. UASS and AF were operated at $37{ }^{\circ} \mathrm{C}$. In the UASS, maize silage was co-digested with straw at an OLR of 


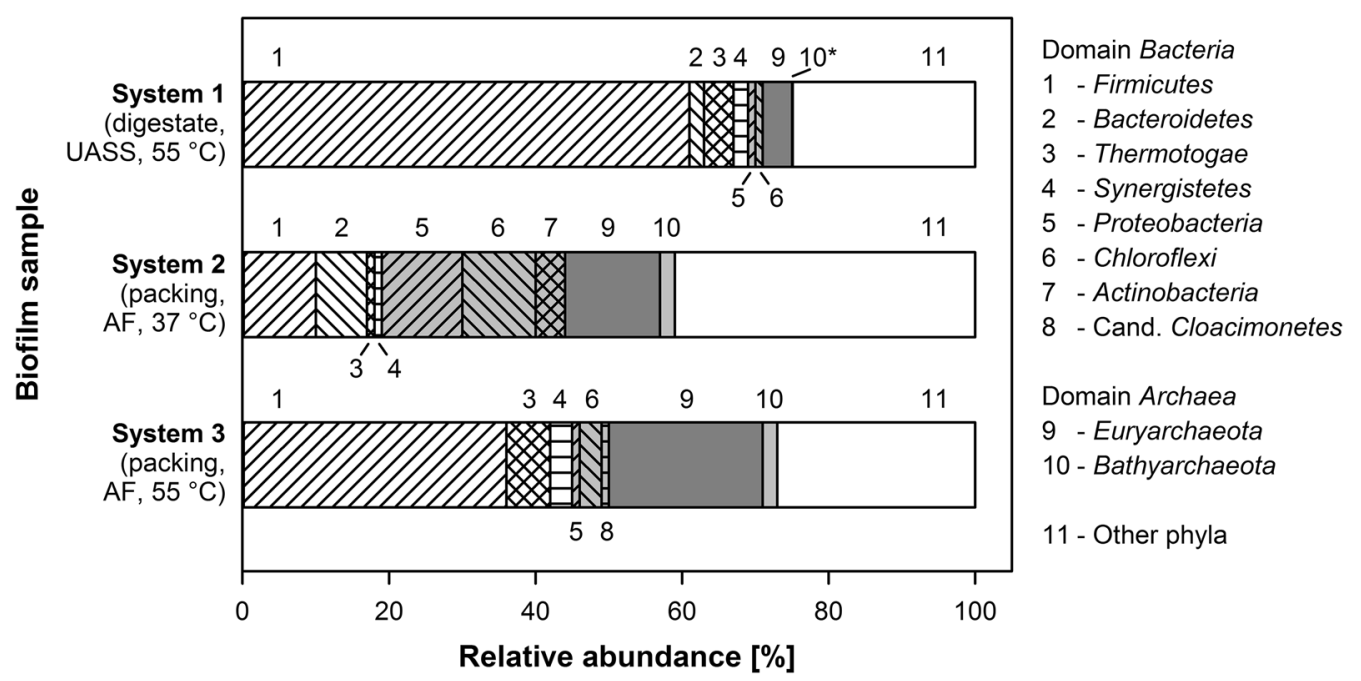

Fig. 1 Relative abundance of the classified phyla present in the biofilms of the sampled thermophilic (systems 1 and 3) and mesophilic (system 2) biogas reactor systems. Analyses were performed on metagenomic data using the LCA (lowest common ancestor) algorithm implemented in MEGAN6 [23]. UASS upflow anaerobic solid-state reactor, AF anaerobic filter; ${ }^{*}$ relative abundance $0.1 \%$

$3.0 \mathrm{~g}_{\mathrm{VS}} \mathrm{L}^{-1} \mathrm{day}^{-1}$. Samples were taken from the methanogenic biofilms on the surfaces of randomly selected polyethylene packings of the AF at day 72 of operation. System 3 was constructed, operated, and sampled similar to system 2 but in this case, the entire system was operated at $55{ }^{\circ} \mathrm{C}$. Further details on reactor operation were provided as Additional file 1.

\section{Metagenome sequencing, assembly, and binning, and functional analyses of obtained MAGs}

Total microbial community DNA was extracted from samples and stored at $-20{ }^{\circ} \mathrm{C}$ using the FastDNA ${ }^{\mathrm{TM}}$ Spin Kit for Soil (MP Biomedicals, USA) according to the manufacturer's instructions. Metagenome shotgun libraries were constructed applying the TruSeq DNA PCR-Free Library Preparation Kit (Illumina) and sequenced on the Illumina MiSeq system utilizing the V2 kit chemistry (Illumina). Trimmed and quality controlled metagenome sequences were assembled with MEGAHIT [20] setting the 'meta-sensitive' option and a minimal contig size of $1000 \mathrm{bp}$. Mappings of the metagenome data sets onto the assemblies were performed applying bbmap from the BBTools package [21] and were further processed with SAMtools [22]. LCAs (lowest common ancestor) of the contigs were computed with MEGAN6 [23] and were used as taxonomic assignments. For abundance determination of the taxonomically assigned contigs, the transcripts per million (TPM) was computed based on the mapped sequencing reads per reactor system individually. Binning of the assemblies was performed on contigs with a minimal coverage of twofold applying MetaBAT with default parameters [24]. Contamination and completeness level of the identified Bathyarchaeota MAGs were assessed with CheckM [25] and acdc [26]. Obtained Bathyarchaeota MAGs were subsequently annotated applying the program Prokka [27] and uploaded into the software platform GenDB [28] for functional analysis. Detailed information on the subsequent bioinformatical analysis of obtained metagenome datasets, i.e., assembly, binning, and functional analysis, is provided as Additional file 1 .

\section{Phylogenetic classification of the determined Bathyarchaeota MAGs in relation to members of the domain Archaea}

To phylogenetically classify the Bathyarchaeota MAGs analyzed in relation to members of the domain Archaea, the phylogenetic trees based on concatenated singlecopy-genes (SCG) and, in addition, on 16S rRNA genes were constructed. The SCG phylogenetic tree was built with 14 MAGs assigned previously to the phylum Bathyarchaeota or to MCG (Additional file 2), respectively, and 128 archaeal genomes selected from IMG/M [29]. The 16S rRNA gene based tree was generated using $16 \mathrm{~S}$ rRNA gene sequences derived from selected archaeal representatives publically available in the SILVA database. Calculation of phylogenetic trees was accomplished applying RAxML version 8.1.16 [30] using the PROTGAMMALGF model with bootstrap calculations based on 1000 replicates and visualized with Phyl.io [31]. Further details are provided as Additional file 1. 
Table 1 Statistics and general features of the Bathyarchaeota MAGs ATB-1, $-2,-3,-4$, and -5 analyzed in this study

\begin{tabular}{|c|c|c|c|c|c|}
\hline $\begin{array}{l}\text { Metagenome- } \\
\text { assembled genome }\end{array}$ & ATB-1 & ATB-2 & ATB-3 & ATB-4 & ATB-5 \\
\hline \multirow[t]{3}{*}{ Origin } & \multicolumn{4}{|c|}{ Thermophilic biogas reactor system $\left(55^{\circ} \mathrm{C}\right)$} & \multirow{2}{*}{$\begin{array}{l}\text { Mesophilic biogas } \\
\text { reactor system }\left(37^{\circ} \mathrm{C}\right) \\
\text { AF of system } 2\end{array}$} \\
\hline & Digestate of system 1 & AF of system 3 & AF of system 3 & AF of system 3 & \\
\hline & $\begin{array}{l}\text { Cellulolytic/ } \\
\text { hydrolytic biofilm }\end{array}$ & $\begin{array}{l}\text { Methanogenic } \\
\text { biofilm }\end{array}$ & $\begin{array}{l}\text { Methanogenic } \\
\text { biofilm }\end{array}$ & $\begin{array}{l}\text { Methanogenic } \\
\text { biofilm }\end{array}$ & Methanogenic biofilm \\
\hline Total length [bp] & $2,038,732$ & $1,574,857$ & $1,495,994$ & $1,083,171$ & $1,914,325$ \\
\hline Number of contigs & 152 & 59 & 131 & 168 & 184 \\
\hline Largest contig & 78,618 & 181,661 & 47,427 & 28,450 & 85,214 \\
\hline N50 & 18,903 & 37,126 & 13,707 & 8072 & 19,864 \\
\hline GC content $[\%]$ & 48.45 & 45.80 & 45.36 & 48.94 & 42.17 \\
\hline Protein-coding genes & 2279 & 1685 & 1709 & 1294 & 2042 \\
\hline Hypothetical proteins & 873 & 597 & 671 & 501 & 832 \\
\hline rRNA genes & 3 (16S-23S-5S) & n.d. & n.d. & n.d. & n.d. \\
\hline tRNA genes & 39 & 34 & 22 & 15 & 27 \\
\hline Completeness $^{\mathrm{a}}$ & 88.79 & 92.06 & 82.28 & 64.83 & 86.30 \\
\hline Contamination $^{\mathrm{a}}$ & 5.61 & 4.28 & 3.74 & 4.43 & 5.14 \\
\hline
\end{tabular}

$A F$ anaerobic filter, n.d. not determined

a Completeness and contamination were estimated by [25]

\section{Results and discussion}

\section{AD biofilm community structure}

In contrast to aqueous process liquids, the surface-associated biofilms in anaerobic biogas reactors were rarely analyzed [32]. In this study, two different thermophilic $\left(55^{\circ} \mathrm{C}\right.$, systems 1 and 3$)$ and one mesophilic $\left(37^{\circ} \mathrm{C}\right.$, system 2) laboratory-scale biogas fermenter systems digesting crop biomass were sampled to determine the presence of Bathyarchaeota members in the microbial biofilms. Due to the respective sampling site, the biofilm sampled from the surface of the digestate of system 1 can be regarded as primarily cellulolytic/hydrolytic and acidogenic but also, although less pronounced, as methanogenic. In contrast, the biofilms established on the surface of the packings in the AFs of systems 2 and 3 are assumed to predominantly represent the methanogenic phase.

To characterize the microbial community compositions in these biofilms, high-throughput whole microbial metagenome sequencing was performed. The three corresponding metagenome datasets generated on the Illumina MiSeq system comprise between 21,963,917 (system 3) and 25,209,139 sequence reads (system 2) (Additional file 3). Taxonomic classification of the biogas biofilm microbiome members based on metagenome sequence data was accomplished as described previously applying the LCA approach on taxonomically assigned contigs. In total 61,633 contigs for system 1, 170,682 contigs for system 2 and 68,904 contigs for system 3 were classified to be of prokaryotic origin; between 1.71 and
$3.66 \%$ sequence reads assembled as contigs remained with no further taxonomic assignment (Additional file 3). For further analysis, metagenome sequences assigned to either the domain Bacteria or Archaea were taken as $100 \%$.

Figure 1 represents relative abundances of classified sequences on phylum level of the analyzed biofilms. On higher taxonomic ranks, all taxonomic profiles showed the dominance of the domain Bacteria representing between 66 and $96 \%$ of all classified metagenome sequences. The most abundant phyla of the bacterial sub-communities in all biofilm samples are the Firmicutes (between 10 and 61\%) followed by Proteobacteria (between 1 and 11\%), Chloroflexi (between 1 and 10\%), and Thermotogae (between 1 and 6\%). The abundance of further phyla such as Synergistetes and Candidatus Cloacimonetes in thermophilic biofilms and Bacteroidetes and Actinobacteria in the mesophilic biofilm is in any case below $10 \%$. As expected, these results support the importance of Firmicutes for anaerobic cellulolysis/ hydrolysis, acidogenesis, and acetogenesis at mesophilic and thermophilic temperatures.

Taxonomic classification of the archaeal sub-communities revealed between 4 and 23\% Archaea (Fig. 1). Members of the phylum Euryarchaeota are abundant in all microbiomes analyzed, representing between $4 \%$ (in the thermophilic cellulolytic/hydrolytic biofilm of system 1) and 21\% (in system 3) of all classified metagenome sequences. Among the archaeal sequences obtained 


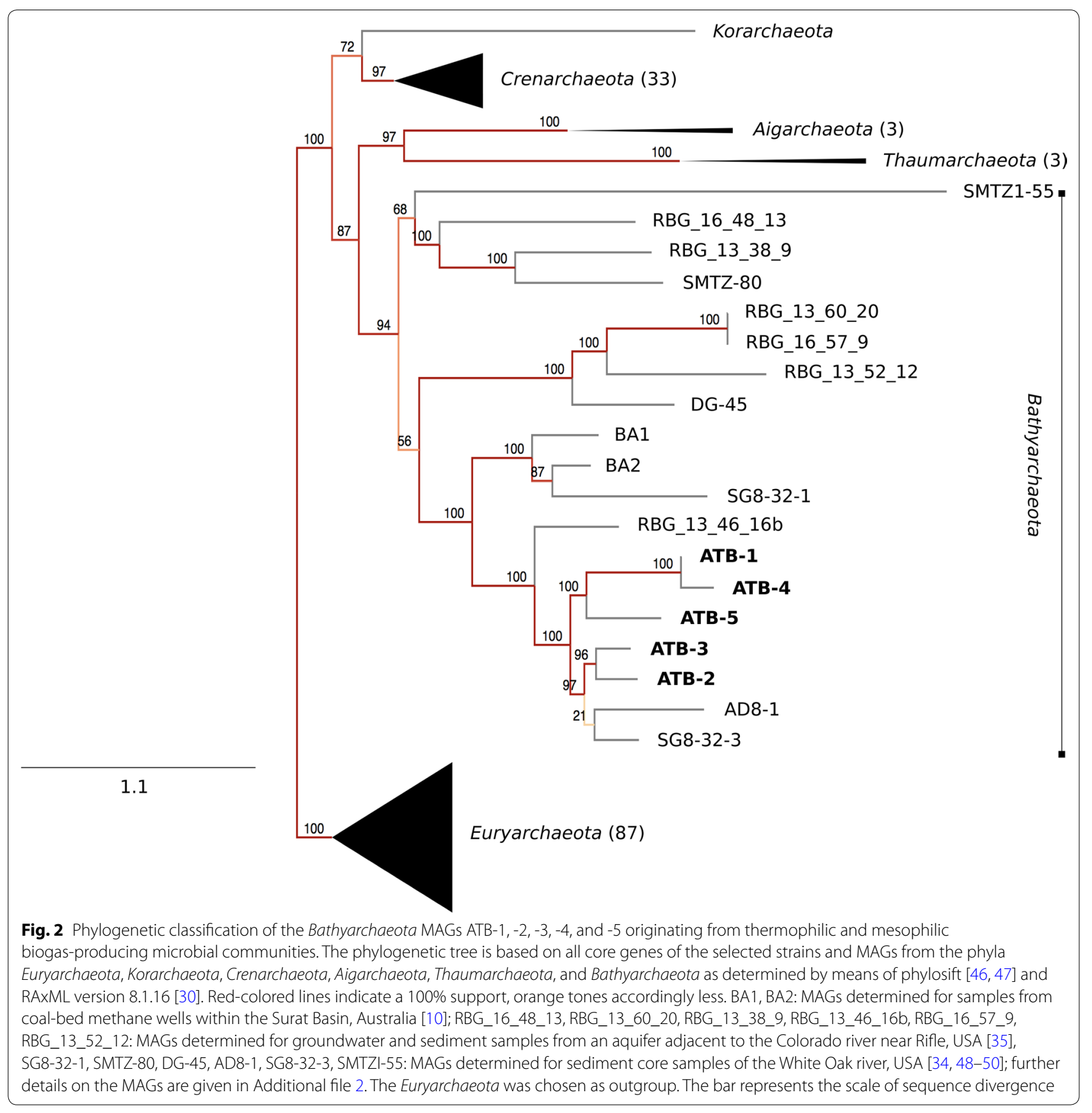

for biofilms of the reactor systems 1, 2 and 3, 0.1, 2, and $2 \%$, respectively, were classified to represent the phylum Bathyarchaeota. This is the first study, in which members of the newly proposed phylum Bathyarchaeota [10] were identified in biotechnological biogas-producing reactor systems digesting crop material.
Phylogenetic affiliation of compiled Bathyarchaeota MAGs To infer genetic potentials and possible functional roles of the detected so far unknown species assigned to the phylum Bathyarchaeota, metagenome assemblies followed by genome binning were applied. This approach enables the identification of new and uncharacterized genomes without the availability of reference database entries. The analysis resulted in the binning of a total of 78 MAGs that met the criteria of a minimum of $50 \%$ genome completeness 


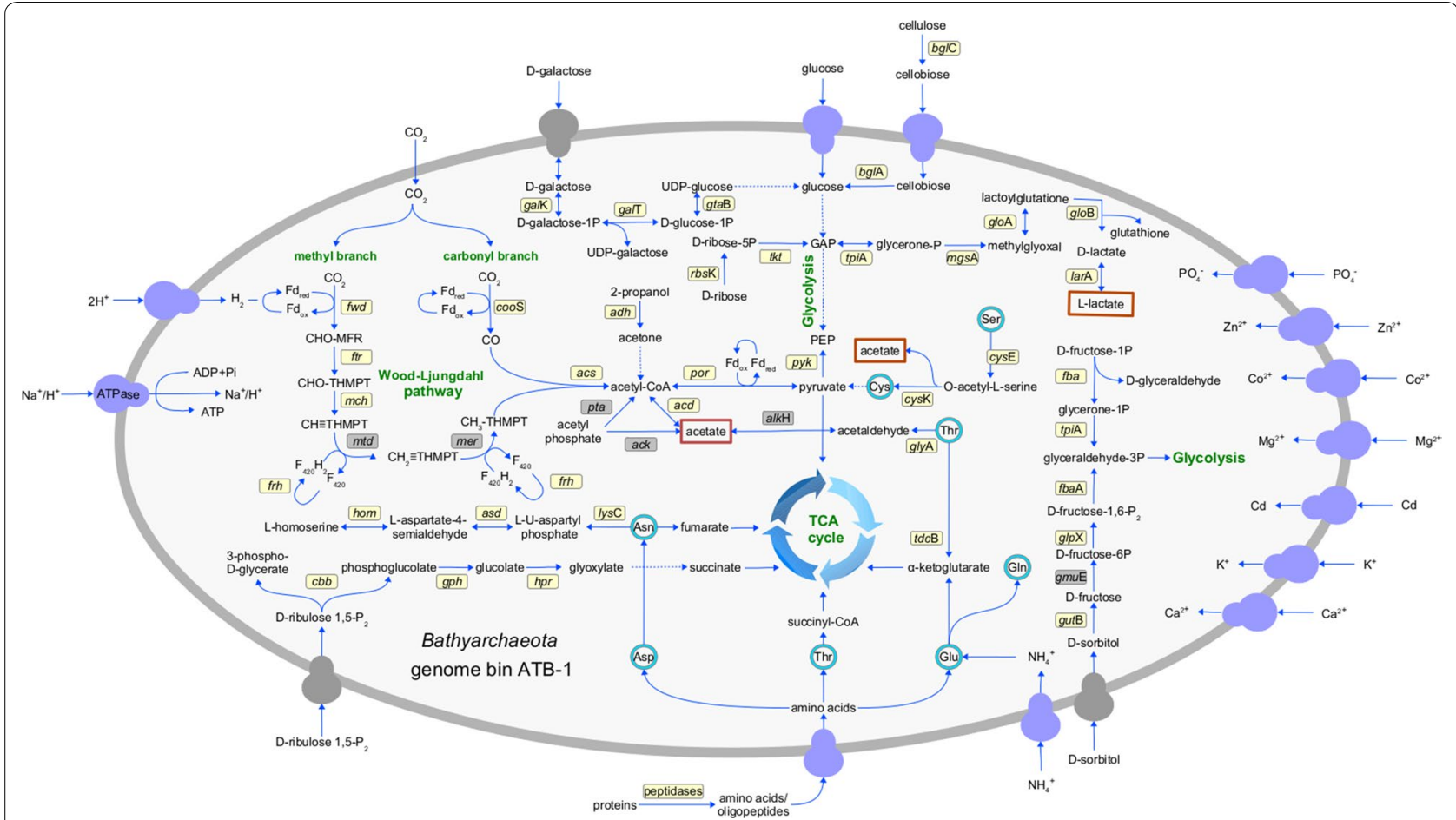

Fig. 3 Metabolic reconstruction of central carbon metabolism pathways in Bathyarchaeota MAG ATB-1. Predicted metabolic pathways for conversion of carbohydrates, proteins and amino acids into acetate, lactate, and tricarboxylic acid intermediates are presented. Determined genes are shown in yellow boxes, determined transport systems are displayed in blue, and missing but presumed transport systems are indicated by gray coloring. Central metabolic pathways are highlighted in green. The identification numbers (locus tags) of MAG ATB-1 genes included in this analysis are listed in Additional file 7

and low contamination rates, i.e., less than 10\%. All MAGs considered (Additional file 4) represent phyla shown in Fig. 1. Five of 78 MAGs belong to the phylum Bathyarchaeota. The MAGs ATB-1 (derived from the system 1 dataset) and ATB-2, -3 , and -4 (system 3 dataset) were obtained for the thermophilic biofilms, and the MAG ATB-5 (system 2 dataset) was determined for the mesophilic biofilm. The MAGs were estimated to be $65-92 \%$ complete as determined by the presence of single-copy marker genes (Table 1). The amount of contamination determined for the MAGs analyzed was low and might be caused by strain heterogeneity. Established MAGs' sizes ranged from 1.1 to $2.0 \mathrm{Mb}$ and featured GC contents from 42.17 to $48.94 \%$. General genome features, e.g., assembly status, size, GC-content, and numbers of predicted genes, are summarized in Table 1.

To determine the phylogenic affiliation of the five MAGs recovered from the metagenome data, SCG encoded gene products were compared to orthologous proteins of other members of the domain Archaea (Fig. 2). The resulting phylogenetic tree showed separation of the analyzed MAGs from other archaeal phyla included in this analysis, namely the Euryarchaeota, Korarchaeota, Crenarchaeota, Aigarchaeota, and
Thaumarchaeota. Furthermore, the position of newly identified MAGs in the phylogenetic tree supports their affiliation to the phylum Bathyarchaeota.

Furthermore, the SCG based phylogenetic tree points to the closer relatedness of MAGs ATB-1 and MAG ATB-4 among the five analyzed MAGs. Hence, average nucleotide sequence identities (ANI) [33], suitable for species demarcation, were calculated between all MAGs analyzed (Additional file 5). MAGs ATB-1 and -4 showed an ANI value of $99.5 \%$, indicating that these two members belong to the same species, whereas the remaining MAGs featured ANI values below 97\% representing the species boundary [33]. However, it must be noted that the MAG ATB- 4 only features a completeness of $65 \%$. Moreover, it represents the smallest Bathyarchaeota MAG among the analyzed bins. Therefore, the statement about its species affiliation remains uncertain.

Interestingly, the Bathyarchaeota MAGs determined in this study cluster with the MAGs AD8-1 and SG832-3 originating from sediment cores of the White Oak river [34]. In contrast, they are separated from the MAGs BA1 and BA2 from a deep aquifer [10], SG8-32-1 (White Oak river habitat, [34] and RBG_13_46_16b (aquifer adjacent to the Colorado river [35]. Together with the 


\begin{tabular}{|c|c|c|c|c|c|c|}
\hline & & \multicolumn{5}{|c|}{ Metagenome-assembled genomes (MAGs) } \\
\hline & & ATB-1 & ATB-2 & ATB-3 & ATB-4 & ATB-5 \\
\hline \multirow{2}{*}{$\begin{array}{c}\text { Endo- and exo-1,4- } \\
\beta \text {-D-glucanase (Cellulase) }\end{array}$} & GH5 & 4 & & 1 & 2 & \\
\hline & GH9 & 1 & & & & \\
\hline \multirow{4}{*}{ Hemicellulase } & GH26 & & & & & 1 \\
\hline & GH30 & & & & & 1 \\
\hline & GH53 & & 1 & & & \\
\hline & GH74 & 4 & & & & \\
\hline \multirow{5}{*}{ Starch and glycogen hydrolase } & GH13 & & & & & 3 \\
\hline & GH15 & & & & & 1 \\
\hline & GH57 & & 1 & 1 & & 2 \\
\hline & GH77 & & & & & 1 \\
\hline & GH133 & 1 & 1 & 1 & 1 & 2 \\
\hline \multirow{4}{*}{$\begin{array}{l}\text { Glycosidase (hydrolysis } \\
\text { of single sugar residues } \\
\text { from non-reducing ends) }\end{array}$} & GH1 & & & 1 & & \\
\hline & $\mathrm{GH} 2$ & 1 & & & 1 & \\
\hline & GH4 & & 2 & & & \\
\hline & GH120 & 1 & 3 & 1 & 3 & \\
\hline Oligosaccharide phosphorylase & GH130 & 3 & & & & \\
\hline \multicolumn{2}{|c|}{ Total no. of hits to CAZy entries } & 79 & 50 & 50 & 43 & 56 \\
\hline
\end{tabular}

Fig. 4 Diversity of genes encoding carbohydrate-active enzymes (CAZymes) predicted to be involved in hydrolysis and/or rearrangement of glycosidic bonds for the Bathyarchaeota MAGs ATB-1,-2,-3,-4, and -5. The screening for the presence of CAZymes was accomplished applying the Hidden-Markov-Model (HMM)-based Carbohydrate-active-enzyme Annotation database dbCAN [37]. The numbers of genes belonging to a corresponding glycosyl hydrolase (GH) family are given in the fields

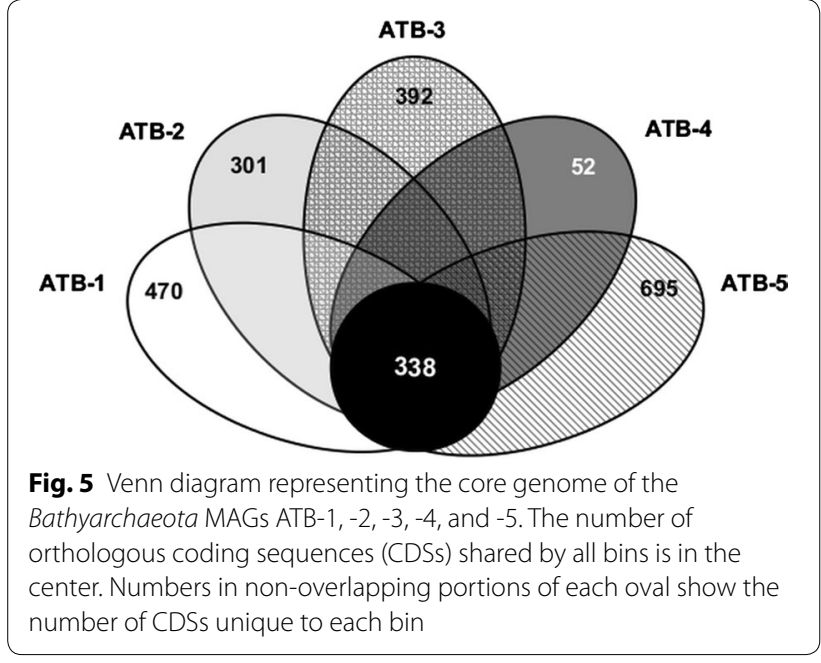

Bathyarchaeota members AD8-1 and SG8-32-3, the MAGs obtained in this study build their own phylogenetic clade and revealed differences to the other recently published MAGs for MCG members. These results were confirmed by a $16 \mathrm{~S}$ rRNA gene-based phylogenetic tree (Additional file 6), computed with sequences of archaeal members from the SILVA database and the 16S rRNA gene sequences from ATB-1.

\section{Pathways for carbohydrate metabolism present in the compiled Bathyarchaeota MAGs}

The five Bathyarchaeota MAGs determined for the microbial biofilms residing in mesophilic and thermophilic biogas reactors were compared using the EDGAR software [36] in order to calculate the set of MAG-specific and shared protein-coding genes. The core genome of the MAGs analyzed appears to be small, including on average less than $26 \%$ of the genes of each MAG. This analysis revealed 338 orthologous genes shared by all of the analyzed MAGs (Fig. 5). These findings illustrate a large degree of genomic diversity in this Bathyarchaeota group. However, taking into account that ATB-4 represents the smallest of the analyzed Bathyarchaeota MAGs (65\% completeness), an overestimation or on the contrary an underestimation of the genetic diversity in this group is most likely. 
To infer the functional roles of Bathyarchaeota MAGs originating from the sampled biofilms of mesophilic and thermophilic biogas reactor systems, metabolic reconstructions were done focusing on fermentation pathways represented in the KEGG database (Additional file 7). In Fig. 3, an overview of the major carbon compound utilizing metabolic pathways is exemplary given for MAG ATB-1, which is the largest MAG determined in this study.

Genomic profiling of the Bathyarchaeota MAGs and identification of genes encoding carbohydrate-active enzymes by means of the CAZy (Carbohydrate-ActiveenZYmes) Database annotation web-server dbCAN [37] showed that all five MAGs have the genetic potential to import and utilize different carbohydrates including cellulose, cellobiose, galactose, glucose, ribose, and, additionally, sorbitol with ATB-1 showing the highest number of hits to CAZy entries (Fig. 4). Decomposition of these compounds results in metabolites that can enter the glycolysis pathway, which is completely encoded in all Bathyarchaeota MAGs analyzed. This indicates a metabolism based on carbohydrate fermentation as it was previously proposed for Bathyarchaeota members originating from other environments [34, 38].

Biomasses such as maize and straw ('energy crops') used for $\mathrm{AD}$ in biogas plants of this study represent plant materials rich in long-chained carbohydrates such as cellulose, hemicellulose, xylan, and starch, among others, but additionally comprise considerable amounts of proteins. Therefore, Bathyarchaeota MAGs were screened for genes encoding enzyme involved in protein, peptide, and amino acid transport and metabolism. The genetic repertoire of the MAGs analyzed also uncovered their potential to utilize proteins and amino acids as growth substrates which is in line with previous findings [10, 34]. In this context, all genes encoding enzymes involved in asparagine, aspartate, alanine, threonine, glutamate, glutamine, serine, and homoserine degradation into tricarboxylic acid (TCA) cycle intermediates and, additionally, pyruvate were identified (Additional file 7). The evidence for genes for carbohydrate, protein, and amino acid uptake and degradation indicate that Bathyarchaeota from the analyzed biogas plant share a heterotrophic metabolism. As it was previously postulated for Bathyarchaeota from the White Oak River sediments [34], this metabolism is primarily based on complex carbohydrates as carbon source augmented by utilization of peptides and amino acids.

Furthermore, the gene repertoire of the Bathyarchaeota MAGs revealed a set of genes, which were assigned to the Wood-Ljungdahl (WL) pathway. This pathway plays an important role in carbon fixation and acetate utilization in acetogens and methanogenesis in methanogenic
Archaea and is characterized by two branches, namely the Western/Carbonyl and the Eastern/Methyl branch [39]. The reaction cascades of both WL branches can proceed in forward and reverse direction, either from carbon dioxide $\left(\mathrm{CO}_{2}\right)$ or carbon monoxide $(\mathrm{CO})$ to acetyl-CoA and further compounds or from acetyl-CoA and its precursors, such as acetate, towards $\mathrm{CO}_{2}$. Acetoclastic methanogens utilize the pathway in reverse direction generating energy by converting acetate to methane $\left(\mathrm{CH}_{4}\right)$ and $\mathrm{CO}_{2}[39,40]$. Hydrogenotrophic methanogens use the Eastern/Methyl branch for methane formation as well as the forward direction of the Western/Carbonyl branch for cell carbon assimilation or acetate generation.

The Western/Carbonyl and the Eastern/Methyl branches of the WL pathway are nearly completely encoded in the Bathyarchaeota MAGs analyzed, with the exception of the genes encoding methylenetetrahydromethanopterin dehydrogenase (Mtd) and 5,10-methylenetetrahydromethanopterin reductase (Mer), which were probably missed by the binning approach. AcetylCoA, produced by enzymatic reactions of the WL pathway, plays an important role in the cell carbon cycle and also feeds into the TCA cycle, the genes of which are encoded in the Bathyarchaeota MAGs. Genes for acetate assimilation mediated by phosphotransacetylase ( $p t a)$ and acetate kinase (ack) needed for conversion of acetylCoA to acetylphosphate and subsequently to acetate were not identified in any of the five MAGs. This is in agreement with previous findings described for the Bathyarchaeota MAGs BA1 and BA2 [10], but is controversial to the findings of He et al. [17] for the MAGs B24, B26-1, and B26-2. However, the acetyl-CoA synthase gene $(a c d)$ involved in acetate formation from acetyl-CoA and vice versa is encoded in all Bathyarchaeota MAGs of this study, with acetate being proposed as possible fermentation end-product (Fig. 3, Additional file 7).

\section{Absence of genes for enzymes involved in methanogenesis in the compiled Bathyarchaeota MAGs}

Since Bathyarchaeota MAGs were recovered from metagenome sequence datasets of biogas-producing biofilms, further genes and pathways playing a role in methane metabolism were analyzed. Neither hydrogenotrophic nor acetoclastic or methylotrophic methanogenesis pathways were completely encoded in the Bathyarchaeota MAGs. Furthermore, the morA gene encoding for methyl-coenzyme $M$ reductase, the key enzyme of the methane production process, is also missing in the five MAGs analyzed, indicating for incapacity of these MAG to produce methane. Additional $m c r$ gene sequence screening in the metagenome datasets leads to the identification of two $m c r$ A gene sequences, showing sequence identity of 93 and $94 \%$ with 
uncultured archaeal clones or Methanoculleus marisnigri, respectively.

However, all MAGs possess complete sets of genes encoding [NiFe] membrane-bound hydrogenase (Ech), cytoplasmic coenzyme $\mathrm{F}_{420}$-reducing [NiFe]-hydrogenase (Frh), and cytoplasmic [NiFe]-hydrogenase (Mvh) needed for activation of $\mathrm{H}_{2}$ during methanogenesis. Moreover, genes encoding heterodisulfide reductase ( $\mathrm{Hdr}$ ) and cytoplasmic [NiFe]-hydrogenase (Mvh) also were identified. Likewise, almost all genes of the $\mathrm{V}$-type $\mathrm{Na}^{+}$/ $\mathrm{H}^{+}$-transporting ATPase (atpABCDEFHIK) were also nearly completely detected in the Bathyarchaeota MAGs. These findings indicate that a membrane-bound electron transport chain potentially enabling energy conservation based on a proton or sodium membrane gradient and an ATPase activity may operate.

\section{Capacities of compiled Bathyarchaeota MAGs to face unfavorable process conditions}

To examine the unique metabolic potential of the five detected Bathyarchaeota MAGs, the MAG-specific gene sets were calculated and classified according to Cluster of Orthologous Groups of proteins (COG) categories (Additional file 8) applying the web server for metagenomic analysis WebMGA [41]. Between 52 (in case of MAG ATB-4) and 695 (in case of MAG ATB-5) singletons were found (Fig. 5). About three quarters of each MAG's unique genes do not correlate to any gene in the COG database.

However, many COG-classified singletons represent genes for proteins participating in amino acid transport and metabolism (E), inorganic ion transport and metabolism (P), or carbohydrate transport and metabolism (G). These functional categories are of importance for $\mathrm{AD}$, since they are primarily connected with plant biomass degradation.

MAG ATB-2, originating from the thermophilic AFpacking-attached methanogenic biofilm of system 3, possesses more classified genes than the other Bathyarchaeota MAGs. Among its 301 singletons are genes coding for 192 hypothetical proteins, but also for a zinc dependent phospholipase, cadmium, cobalt, and zinc antiporters, and a potassium proton pump. Hence, phospholipid degradation might play a role for the Bathyarchaeota taxon represented by MAG ATB- 2 . The presence of the potassium transporter might be involved in compensation of osmotic stress as supposed for the methanogenic archaeon Methanoculleus bourgensis $\mathrm{MS}^{\mathrm{T}}{ }^{\text {[42] }}$.

Among the other Bathyarchaeota MAGs, ATB-5 possesses many classified singletons (61\%), representing those genetic determinants that may specify characteristic features of this MAG. These 695 MAG-specific genes encode proteins involved in transport of the amino acids leucine, isoleucine, and valine. Furthermore, genes encoding proteins for trehalose utilization as carbon or energy source and lactate synthesis mediated by lactate dehydrogenase were also identified.

Transport of ions and nutrients is of importance for microorganisms as reflected by the wide variety of encoded enzymatic pathways. Hence, the supply of anaerobic digesters converting crop material with trace elements is crucial [43]. The Bathyarchaeota MAGs determined in this study were screened for their coding capacity regarding transport systems for inorganic and metal ions and other compounds. Genes encoding transport systems for calcium, potassium, cadmium, magnesium, cobalt, zinc, and phosphate were identified (Additional file 7).

Furthermore, a gene encoding the archaeal-specific ammonium $\left(\mathrm{NH}_{4}^{+}\right)$transporter (amt), also known from the euryarchaeon Archaeoglobus fulgidus [44], was identified in all MAGs except for the MAG ATB-4. $\mathrm{NH}_{4}{ }^{+}$can be assimilated directly by glutamine synthetase (GS) and glutamate synthase (GOGAT) into glutamine and glutamate, respectively. The genes encoding these enzymes are present in all five analyzed Bathyarchaeota MAGs.

Analysis of the Bathyarchaeota MAGs revealed also several genes of the glyoxalase metabolism, a common pathway involved in the conversation of the toxic glycolytic byproduct methylglyoxal to D-lactate [45]. First, the glycolysis intermediate glycerone phosphate is converted to methylglyoxal by the methylglyoxal synthase (Mgs) and subsequently to the thioester S-D-lactoyltrypanothione via the enzyme glyoxalase-I (GloA). In the second step, glyoxalase-II (GloB) catalyzes hydrolysis of this thioester, releasing D-lactate. Genes encoding all three enzymes were only identified in the MAGs ATB-1, -2 , -3 , and -4 , whereas the remaining bin ATB- 5 does not encode the methylglyoxal synthase (Mgs) involved in the first reaction step of the glyoxalase metabolism.

MAG ATB-1 was the only one harboring genes of the Clustered Regularly Interspaced Short Palindromic Repeats (CRISPR) cas system, an adaptive microbial immune system that provides resistance against invasion of phages and mobile elements. In the MAG ATB1 , nine cas genes of type I-A were identified, which are located in direct vicinity to the CRISPR sequences (data not shown). The CRISPR array is composed of ten 37-bpdirect-repeats and nine spacers of $39 \mathrm{bp}$. The presence of CRISPR systems in Bathyarchaeota is in line with previously published findings indicating that Archaea may deal with foreign-DNA infections in its habitat, e.g., phages [42].

Additionally, to identify unique genes, present only in Bathyarchaeota members originating from biogas reactor environments, the core genome of the MAGs ATB-1 
to 5 was compared with the pan genome of fourteen other Bathyarchaeota MAGs (for details see Fig. 2 and Additional file 2) using the program EDGAR. In total, 17 unique genes, also called singletons, were identified for the group of biogas Bathyarchaeota indicating that biogas biofilm Bathyarchaeota are not characterized by specific capabilities. The unique genes of Bathyarchaeota MAGs from biogas systems encode eight hypothetical proteins as well as enzymes of the amino acid synthesis metabolism.

\section{Conclusions}

In contrast to the Bathyarchaeota detected in coal-bed methane wells [10], the Bathyarchaeota in the analyzed biogas reactor biofilms are not able to produce methane via the hitherto known methanogenesis pathway. However, the reconstruction of the metabolic pathways suggests that the analyzed Bathyarchaeota may base their metabolisms on carbohydrates and amino acids utilization as well as on $\mathrm{CO}_{2}$ fixation. Genes for extracellular hydrolysis of cellulose but also extracellular peptidases with corresponding transporter systems were found. Acetate and lactate were predicted as possible end-products of the fermentation process. Based on these findings, the analyzed MAGs were predicted to represent hydrolytic and eventually also cellulolytic and proteolytic Archaea involved in hydrogenesis and acidogenesis within the AD and biomethanation process. Due to their presence in biofilms, also a syntrophic co-operation with methanogenic Euryarchaeota could be possible. This is an outstanding finding for members of the domain Archaea, since only bacterial microorganisms were previously thought to be involved in the anaerobic biomass degradation in biogas reactor systems.

This study initiates rethinking of the task sharing between Bacteria and Archaea regarding successive decomposition of macromolecular compounds. Future work has to show whether findings obtained for laboratory-scale biogas reactors can be biotechnologically exploited by applying Bathyarchaeota species in industrial-scale biomass conversion processes. Accordingly, it is important to determine the occurrence of Bathyarchaeota members in industrial, i.e., productionscale biogas plants. In particular, correlations of their abundances with the utilization of specific substrates or particular reactor characteristics and conditions should be uncovered. Continuative studies will certainly benefit from the comprehensive genomic information on Bathyarchaeota members from biogas reactor systems by integrating this knowledge into models describing interactions within complex AD communities.

\section{Additional files}

Additional file 1. Supporting information on materials and methods.

Additional file 2. Previously published MAGs for the phylum Bathyarchaeota. The phylogenetic affiliation is shown in Fig. 2. *, completeness and contamination were estimated by CheckM [25].

Additional file 3. Sequencing and assembly statistics of the metagenome data for the microbial communities of the thermophilic and mesophilic biogas reactor systems. UASS, upflow anaerobic solid-state reactor; AF, anaerobic filter; n.d., not determined.

Additional file 4. Compiled metagenome-assembled genomes that met the criteria of a minimum of $50 \%$ genome completeness and contamination rates of $10 \%$.

Additional file 5. The average nucleotide identity (ANI) analysis of the analyzed Bathyarchaeota MAGs.

Additional file 6. Phylogenetic position of the Bathyarchaeota MAG ATB-1 within the selected archaeal representatives available in the SILVA database. The scale bar below the tree represents sequence divergence.

Additional file 7. Genomic loci of the analyzed Bathyarchaeota MAGs encoding those enzymatic functions, which are mentioned in the metabolic scheme (Fig. 3) as well as in the main text. n.d., not determined.

Additional file 8. Categorization of analyzed Bathyarchaeota MAGs' unique genes according to Clusters of Orthologous Groups of proteins (COGs).

\section{Abbreviations}

AD: anaerobic digestion; AF: anaerobic filter; BGP: biogas plant; MAG: metagenome-assembled genomes; CAZymes: carbohydrate-active enzymes; GH: glycosyl hydrolase; LCA: lowest common ancestor; OLR: organic loading rate; UASS: upflow anaerobic solid-state reactor; VS: volatile substances.

\section{Authors' contributions}

IM performed the annotation of the MAGs, predicted archaeal fermentation pathways based on MAG sequence information, performed the comparative MAG analyses, coordinated drafting, and drafted corresponding parts of the manuscript. MR carried out the taxonomic classification of the microbial communities, performed the metagenome assembly and binning, determined the phylogenetic relationship between the MAGs, contributed to the results and discussion section, and revised the manuscript. IB, KH, MP, and EN set up, performed, and sampled the anaerobic digestion experiments, and revised the manuscript. IB, KH, and EN performed pre-analyses of microbial DNA samples. SJ participated in bioinformatic data analysis, and revised the manuscript. JB participated in comparative MAG analyses, and revised the manuscript. AP participated in the design of this study, contributed to the results and discussion section, and revised the manuscript. AS, AScz, and MK conceived the study, participated in manuscript coordination, drafted parts of the manuscript, supervised all biological and bioinformatic data analyses, and revised the manuscript. All authors read and approved the final manuscript.

\footnotetext{
Author details

${ }^{1}$ Dept. Bioengineering, Leibniz Institute for Agricultural Engineering and Bioeconomy (ATB), Max-Eyth-Allee 100, 14469 Potsdam, Germany. ${ }^{2}$ Center for Biotechnology (CeBiTec), Bielefeld University, Universitätsstrasse 27, 33615 Bielefeld, Germany. ${ }^{3}$ Computational Metagenomics, Faculty of Technology, Bielefeld University, Universitätsstrasse 25, 33615 Bielefeld, Germany. ${ }^{4}$ Biochemical Conversion Department, Deutsches Biomasseforschungszentrum gemeinnützige $\mathrm{GmbH}$, Torgauer Straße 116, 04347 Leipzig, Germany. ${ }^{5}$ Urban Water Management and Environmental Engineering, Faculty of Civil and Environmental Engineering, Ruhr University Bochum, Universitätsstraße 150, 44780 Bochum, Germany. ${ }^{6}$ Dept. Bioinformatics and Systems Biology, JustusLiebig University Gießen, Heinrich-Buff-Ring 58, 35392 Giessen, Germany.
} 


\section{Acknowledgements}

The authors thank the Fachagentur für Nachwachsende Rohstoffe (FNR) and Projektträger Jülich (PTJ) for their valuable support in project management.

\section{Competing interests}

The authors declare that they have no competing interests.

\section{Availability of data and materials}

The Bathyarchaeota MAG sequences as well as the metagenome sequence data supporting the conclusions of this article are available in the European Nucleotide Archive (ENA) under the Bioproject Accession Number ID PRJEB21266.

\section{Consent for publication}

Not applicable.

\section{Declarations}

Not applicable.

\section{Ethical approval and consent to participate}

Not applicable.

\section{Funding}

This work was supported by the German Federal Ministry of Food and Agriculture (BMEL), Grant Number 22016308, and the German Federal Ministry of Education and Research (BMBF), Grant Number 03SF0381. MR's contribution has been made possible through the German-Canadian DFG international research training group "Computational Methods for the Analysis of the Diversity and Dynamics of Genomes (DiDy)," Grant Number GRK 1906/1.

\section{Publisher's Note}

Springer Nature remains neutral with regard to jurisdictional claims in published maps and institutional affiliations.

\section{Received: 16 January 2018 Accepted: 1 June 2018}

Published online: 19 June 2018

\section{References}

1. Hanreich A, Schimpf U, Zakrzewski M, Schlüter A, Benndorf D, Heyer R, et al. Metagenome and metaproteome analyses of microbial communities in mesophilic biogas-producing anaerobic batch fermentations indicate concerted plant carbohydrate degradation. Syst Appl Microbiol. 2013;36:330-8.

2. Goux X, Calusinska M, Lemaigre S, Klocke M, Udelhoven T, Benizri E, et al. Microbial community dynamics in replicate anaerobic digesters exposed sequentially to increasing organic loading rate, acidosis, and process recovery. Biotechnol Biofuels. 2015;8:122.

3. Stolze Y, Zakrzewski M, Maus I, Eikmeyer F, Jaenicke S, Rottmann N, et al. Comparative metagenomics of biogas-producing microbial communities from production-scale biogas plants operating under wet or dry fermentation conditions. Biotechnol Biofuels. 2015:8:14.

4. Maus I, Koeck DE, Cibis K, Hahnke S, Kim Y, Langer T, et al. Unraveling the microbiome of a thermophilic biogas plant by metagenome and metatranscriptome analysis complemented by characterization of bacterial and archaeal isolates. Biotechnol Biofuels. 2016;9:171.

5. Nettmann E, Bergmann I, Pramschüfer S, Mundt K, Plogsties V, Herrmann C, et al. Polyphasic analyses of methanogenic archaeal communities in agricultural biogas plants. Appl Environ Microbiol. 2010;76:2540-8.

6. Klocke M, Mähnert P, Mundt K, Souidi K, Linke B. Microbial community analysis of a biogas-producing completely stirred tank reactor fed continuously with fodder beet silage as mono-substrate. Syst Appl Microbiol. 2007:30:139-51.

7. Ortseifen V, Stolze Y, Maus I, Sczyrba A, Bremges A, Albaum SP, et al. An integrated metagenome and -proteome analysis of the microbial community residing in a biogas production plant. J Biotechnol. 2016;231:268-79.

8. Stolze Y, Bremges A, Rumming M, Henke C, Maus I, Pühler A, et al. Identification and genome reconstruction of abundant distinct taxa in microbiomes from one thermophilic and three mesophilic productionscale biogas plants. Biotechnol Biofuels. 2016;9:156.

9. Treu L, Kougias PG, Campanaro S, Bassani I, Angelidaki I. Deeper insight into the structure of the anaerobic digestion microbial community; the biogas microbiome database is expanded with 157 new genomes. Bioresour Technol. 2016;216:260-6.

10. Evans PN, Parks DH, Chadwick GL, Robbins SJ, Orphan VJ, Golding SD, et al. Methane metabolism in the archaeal phylum Bathyarchaeota revealed by genome-centric metagenomics. Science. 2015;350:434-8.

11. Gagen EJ, Huber H, Meador T, Hinrichs KU, Thomm M. Novel cultivationbased approach to understanding the miscellaneous crenarchaeotic group (MCG) archaea from sedimentary ecosystems. Appl Environ Microbiol. 2013;79:6400-6.

12. Lazar CS, Biddle JF, Meador TB, Blair N, Hinrichs KU, Teske AP. Environmental controls on intragroup diversity of the uncultured benthic archaea of the miscellaneous Crenarchaeotal group lineage naturally enriched in anoxic sediments of the White Oak River estuary (North Carolina, USA). Environ Microbiol. 2015;17:2228-38.

13. McKay LJ, Hatzenpichler R, Inskeep WP, Fields MW. Occurrence and expression of novel methyl-coenzyme $M$ reductase gene (mcrA) variants in hot spring sediments. Sci Rep. 2017;7:7252.

14. Lloyd KG, Alperin MJ, Teske A. Environmental evidence for net methane production and oxidation in putative ANaerobic MEthanotrophic (ANME) archaea. Environ Microbiol. 2011;13:2548-64.

15. Kubo K, Lloyd KG, Biddle JF, Amann R, Teske A, Knittel K. Archaea of the miscellaneous Crenarchaeotal Group are abundant, diverse and widespread in marine sediments. ISME J. 2012;6:1949-65.

16. Seyler LM, McGuinness LM, Kerkhof LJ. Crenarchaeal heterotrophy in salt marsh sediments. ISME J. 2014;8:1534-43.

17. He Y, Li M, Perumal V, Feng X, Fang J, Xie J, et al. Genomic and enzymatic evidence for acetogenesis among multiple lineages of the archaeal phylum Bathyarchaeota widespread in marine sediments. Nat Microbiol. 2016;1:16035.

18. Pohl M, Heeg K, Mumme J. Anaerobic digestion of wheat strawperformance of continuous solid-state digestion. Bioresour Technol. 2013;146:408-15.

19. Bergmann I, Klocke M. Biofilms in biogas fermenters-community structure, influence on biogas yields and optimization of technical solutions for retaining the microbial biomass (BIOGAS-BIOFILM). Bornimer Agrartechnische Berichte. 2015;87:1-164.

20. Li D, Luo R, Liu CM, Leung CM, Ting HF, Sadakane K, et al. MEGAHIT v1. 0: a fast and scalable metagenome assembler driven by advanced methodologies and community practices. Methods. 2016;102:3-11.

21. DOE Joint Genome Institute (JGI). BBTools; 2014. http://jgi.doe.gov/dataand-tools/bbtools/. Accessed 23 June 2016.

22. Li H, Handsaker B, Wysoker A, Fennell T, Ruan J, Homer N, et al. 1000 Genome Project Data Processing Subgroup, 2009. The sequence alignment/map format and SAMtools. Bioinformatics. 2009;25:2078-9.

23. Huson DH, Beier S, Flade I, Górska A, El-Hadidi M, Mitra S, et al. MEGAN community edition-interactive exploration and analysis of large-scale microbiome sequencing data. PLoS Comput Biol. 2016;12:e1004957.

24. Kang DD, Froula J, Egan R, Wang Z. MetaBAT, an efficient tool for accurately reconstructing single genomes from complex microbial communities. Peer J. 2015;3:e1165.

25. Parks DH, Imelfort M, Skennerton CT, Hugenholtz P, Tyson GW. CheckM: assessing the quality of microbial genomes recovered from isolates, single cells, and metagenomes. Genome Res. 2015;25:1043-55.

26. Lux M, Krüger J, Rinke C, Maus I, Schlüter A, Woyke T, et al. Acdc-automated contamination detection and confidence estimation for singlecell genome data. BMC Bioinformatics. 2016;17:543.

27. Seemann T. Prokka: rapid prokaryotic genome annotation. Bioinformatics. 2014;30:2068-9.

28. Meyer F, Goesmann A, McHardy AC, Bartels D, Bekel T, Clausen J, et al. GenDB-an open source genome annotation system for prokaryote genomes. Nucleic Acids Res. 2003;31:2187-95.

29. Markowitz VM, Chen IM, Chu K, Szeto E, Palaniappan K, Pillay M, et al. IMG/M 4 version of the integrated metagenome comparative analysis system. Nucleic Acids Res. 2014;42:D568-73.

30. Stamatakis A. RAxML version 8: a tool for phylogenetic analysis and postanalysis of large phylogenies. Bioinformatics. 2014;30:1312-3. 
31. Robinson O, Dylus D, Dessimoz C. Phylo.io: interactive viewing and comparison of large phylogenetic trees on the web. Mol Bio Evol. 2016;33:2163-6.

32. Rademacher A, Zakrzewski M, Schlüter A, Schönberg M, Szczepanowski R, Goesmann A, et al. Characterization of microbial biofilms in a thermophilic biogas system by high-throughput metagenome sequencing. FEMS Microbiol Ecol. 2012;79:785-99.

33. Konstantinidis KT, Tiedje JM. Genomic insights that advance the species definition for prokaryotes. Proc Natl Acad Sci USA. 2005;102:2567-72.

34. Lazar CS, Baker BJ, Seitz K, Hyde AS, Dick GJ, Hinrichs KU, et al. Genomic evidence for distinct carbon substrate preferences and ecological niches of Bathyarchaeota in estuarine sediments. Environ Microbiol. 2016;18:1200-11.

35. Anantharaman K, Brown CT, Hug LA, Sharon I, Castelle CJ, Probst AJ, et al. Thousands of microbial genomes shed light on interconnected biogeochemical processes in an aquifer system. Nat Commun. 2016;7:13219.

36. Blom J, Kreis J, Spänig S, Juhre T, Bertelli C, Ernst C, et al. EDGAR 2.0: an enhanced software platform for comparative gene content analyses. Nucleic Acids Res. 2016;44:W22-8.

37. Yin Y, Mao X, Yang J, Chen X, Mao F, Xu Y. dbCAN: a web resource for automated carbohydrate-active enzyme annotation. Nucleic Acids Res. 2012:40:W445-51

38. Lloyd KG, Schreiber L, Petersen DG, Kjeldsen KU, Lever MA, Stehen AD, et al. Predominant archaea in marine sediments degrade detrital proteins. Nature. 2013:496:215-8.

39. Ragsdale SW. Enzymology of the Wood-Ljungdahl pathway of acetogenesis. Ann NY Acad Sci. 2008;1125:129-36.

40. Borrel G, Panagiotis S, Gribaldo A, Gribaldo S. Methanogenesis and the Wood-Ljungdahl pathway: an ancient, versatile, and fragile association. Genome Biol Evol. 2016;8:1706-11.

41. Wu S, Zhu Z, Fu L, Niu L, Li W. WebMGA: a customizable web server for fast metagenomic sequence analysis. BMC Genomics. 2011;12:444.

42. Maus I, Wibberg D, Stantscheff R, Stolze Y, Blom J, Eikmeyer FG, et al. Insights into the annotated genome sequence of Methanoculleus bourgensis MS2 (T), related to dominant methanogens in biogas-producing plants. J Biotechnol. 2015;201:43-53.

43. Demirel B, Scherer P. Trace element requirements of agricultural biogas digesters during biological conversion of renewable biomass to methane. Biomass Bioenerg. 2009;35:992-8.

44. Andrade SL, Dickmanns A, Ficner R, Einsle O. Expression, purification and crystallization of the ammonium transporter Amt-1 from Archaeoglobus fulgidus. Acta Crystallogr Sect F Struct Biol Cryst Commun. 2005;61:861-3.

45. Greig N, Wyllie S, Patterson S, Fairlamb AH. A comparative study of methylglyoxal metabolism in trypanosomatids. FEBS J. 2009;276:376-86.
46. Darling AE, Jospin G, Lowe E, Matsen FA, Bik HM, Eisen JA. PhyloSift: phylogenetic analysis of genomes and metagenomes. PeerJ. 2014;2:e243.

47. Edgar RC. MUSCLE: multiple sequence alignment with high accuracy and high throughput. Nucleic Acids Res. 2004;32:1792-7.

48. Bird JT, Baker BJ, Probst AJ, Podar M, Lloyd KG. Culture independent genomic comparisons reveal environmental adaptations for Altiarchaeales. Front Microbiol. 2016;7:1221.

49. Seitz KW, Lazar CS, Hinrichs KU, Teske AP, Baker BJ. Genomic reconstruction of a novel, deeply branched sediment archaeal phylum with pathways for acetogenesis and sulfur reduction. ISME J. 2016;10:1696-705.

50. Zaremba-Niedzwiedzka K, Caceres EF, Saw JH, Bäckström D, Juzokaite $L$, Vancaester E, et al. Asgard Archaea illuminate the origin of eukaryotic cellular complexity. Nature. 2016;541:353-8.

51. Bolger AM, Lohse M, Usadel B. Trimmomatic: a flexible trimmer for Illumina sequence data. Bioinformatics. 2014;30:2114-212.

52. Hyatt D, Chen GL, LoCascio PF, Land ML, Larimer FW. Hauser LJ. Prodigal: prokaryotic gene recognition and translation initiation site identification. BMC Bioinform. 2015:11:119.

53. Buchfink B, Xie C, Huson DH. Fast and sensitive protein alignment using DIAMOND. Nat Methods. 2015:12:59-60.

54. NCBI Resource Coordinators. Database resources of the national center for biotechnology information. Nucleic Acids Res. 2016;44:D7-19.

55. Bankevich A, Nurk S, Antipov D, Gurevich AA, Dvorkin M, Kulikov AS, et al. SPAdes: a new genome assembly algorithm and its applications to single-cell sequencing. J Comput Biol. 2012;19:455-77.

56. Gurevich A, Saveliev V, Vyahhi N, Tesler G. QUAST: quality assessment tool for genome assemblies. Bioinformatics. 2013;29:1072-5.

57. Lagesen K, Hallin P, Rødland EA, Stærfeldt HH, Rognes T, Ussery DW. RNAmmer: consistent and rapid annotation of ribosomal RNA genes. Nucleic Acids Res. 2007;35:3100-8

58. Tatusov RL, Galperin MY, Natale DA, Koonin EV. The COG database: a tool for genome-scale analysis of protein functions and evolution. Nucleic Acids Res. 2000:28:33-6.

59. Tatusov RL, Natale DA, Garkavtsev IV, Tatusova TA, Shankavaram UT, Rao BS. The COG database: new developments in phylogenetic classification of proteins from complete genomes. Nucleic Acids Res. 2001;29:22-8.

60. Grissa I, Vergnaud G, Pourcel C. The CRISPRdb database and tools to display CRISPRs and to generate dictionaries of spacers and repeats. BMC Bioinform. 2007;8:172.

61. Tomazetto G, Hahnke S, Koeck DE, Wibberg D, Maus I, Pühler A, et al. Complete genome analysis of Clostridium bornimense strain M2/40(T): a new acidogenic Clostridium species isolated from a mesophilic twophase laboratory-scale biogas reactor. J Biotechnol. 2015;232:38-49.
Ready to submit your research? Choose BMC and benefit from:

- fast, convenient online submission

- thorough peer review by experienced researchers in your field

- rapid publication on acceptance

- support for research data, including large and complex data types

- gold Open Access which fosters wider collaboration and increased citations

- maximum visibility for your research: over $100 \mathrm{M}$ website views per year

At BMC, research is always in progress.

Learn more biomedcentral.com/submissions 